\title{
WENDROFF TYPE INEQUALITIES
}

\author{
R. P. SHASTRI AND D. Y. KASTURE
}

\begin{abstract}
Results (1) and (3) of Wendroff on two-variable scalar integral inequalities, quoted without proof by Beckenback and Bellman [1, p. 154] have been generalized to cover a system of integral inequalities by following an approach different from that of Jagdish Chandra and Davis [3].
\end{abstract}

In October 1976 Jagdish Chandra and Davis [3] published their results on the generalization of the Gronwall inequality to cover a system of $n$ integral inequalities in $m$ independent variables. At about the same time we prepared a paper in which we obtained the result in Corollary 1 in their paper by following the approach of differential analysis as in Snow [5]. Although our approach requires the hypothesis of differentiability not needed in [3], it is a constructive approach and it has a potential for being applicable to a large class of differential and integral inequalities. To illustrate this approach, we give a theorem from our unpublished paper which is a generalization of the results (1) and (3) of Wendroff, Beckenback and Bellman [1]. An application of our result is also mentioned.

THEOREM. Let $\phi(x, y)$ be a continuous, nonnegative, $n$-vector function on a two dimensional domain $D$ and $A(x, y), B(x, y), H(x, y)$ be continuous, nonnegative, symmetric $n \times n$ matrix functions with $A(x, y), B(x, y)$ continuously differentiable in $x$ and $y$ and nonincreasing in $y$ and $x$ respectively. If $C$ is any nonnegative constant $n$-vector and $\phi(x, y)$ satisfies

$$
\begin{aligned}
\phi(x, y) \leqslant & C+\int_{0}^{x} A(s, y) \phi(s, y) d s+\int_{0}^{y} B(x, t) \phi(x, t) d t \\
& +\int_{0}^{x} \int_{0}^{y} H(s, t) \phi(s, t) d s d t
\end{aligned}
$$

then

$$
\begin{aligned}
\phi(x, y) \leqslant C^{T} \exp [ & \int_{0}^{x} A(\alpha, y) d \alpha+\int_{0}^{y} B(x, \beta) d \beta \\
& \left.+\int_{0}^{x} \int_{0}^{y}\{A(\alpha, \beta) B(\alpha, \beta)+H(\alpha, \beta)\} d \alpha d \beta\right],
\end{aligned}
$$

where $C^{T}$ is the row vector.

Received by the editors July 29, 1977.

AMS (MOS) subject classifications (1970). Primary 35B45, 35L15; Secondary 34A40, 45D05, 45 F05.

Key words and phrases. Wendroff's inequality, integral inequality, differential inequality, integral equation.

○ American Mathematical Society 1978 
Proof. Let $u(x, y)$ be the solution of the integral equation corresponding to (1). The existence of $u$ can be proved by using the contraction mapping principle, as in Ghoshal and Masood [2 (see Lemma 1)]. Then by Theorem IIIb, p. 130 of Walter [6], we have

$$
\phi(x, y) \leqslant u(x, y) .
$$

Differentiating $u(s, t)$ twice, we obtain

$$
L u=u_{s t}-B u_{s}-A u_{t}-\left(A_{t}+B_{s}+H\right) u=0 .
$$

The equality (4) can be integrated using Riemann's method. For any twice continuously differentiable matrix function $W(s, t ; x, y)$, we have as in Snow [5], (notation as in the figure)

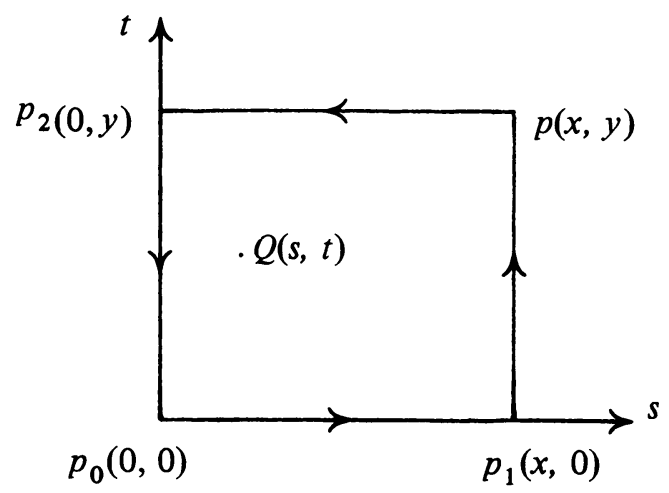

$$
\begin{aligned}
W^{T}(p) u(p) & =u^{T}\left(p_{0}\right) W\left(p_{0}\right)+\int_{p_{2}}^{p}\left[W^{T} A+W_{s}^{T}\right] u d s \\
& +\int_{p_{1}}^{p} u^{T}\left[B W+W_{t}\right] d t+\int_{0}^{x} \int_{0}^{y}\left[W^{T} L u-u^{T} M W\right] d s d t
\end{aligned}
$$

where $M$, defined by

$$
M W=W_{s t}+B W_{s}+A W_{t}-H W
$$

is the adjoint of the operator $L$.

We choose $W$ so that

(i) $W(s, t ; x, y)>0$ and $M W \geqslant 0,0 \leqslant s \leqslant x, 0 \leqslant t \leqslant y$,

(ii) $W^{T} A+W_{s}^{T} \leqslant 0,0 \leqslant s \leqslant x, t=y$,

(iii) $B W+W_{t} \leqslant 0,0 \leqslant t \leqslant y, s=x$.

Then (4) and (5) imply that

$$
W^{T}(p) u(p) \leqslant u^{T}\left(p_{0}\right) W\left(p_{0}\right) .
$$

A function $W(s, t ; x, y)$ satisfying all the requirements (i), (ii) and (iii) is easily obtained. It is 


$$
\begin{aligned}
W(s, t ; x, y)=\exp [ & \int_{s}^{x} A(\alpha, t) d \alpha+\int_{t}^{y} B(s, \beta) d \beta \\
& \left.+\int_{s}^{x} \int_{t}^{y}\{A(\alpha, \beta) B(\alpha, \beta)+H(\alpha, \beta)\} d \alpha d \beta\right] .
\end{aligned}
$$

The desired conclusion now follows from (6) and (3).

The theorem can be applied to prove the uniqueness of the solution of the scalar hyperbolic differential equation

$$
\phi_{x y}=\{a(x, y) \phi(x, y)\}_{y}+\{b(x, y) \phi(x, y)\}_{x}+h(x, y) f(x, y, \phi),
$$

with conditions prescribed on $x=0, y=0$ in such a way that the problem is equivalent to the integral equation

$$
\begin{aligned}
\phi(x, y)= & C+\int_{0}^{x} a(s, y) \phi(s, y) d s+\int_{0}^{y} b(x, t) \phi(x, t) d t \\
& +\int_{0}^{x} \int_{0}^{y} h(s, t) f(s, t, \phi(s, t)) d s d t
\end{aligned}
$$

where $C$ is a nonnegative constant.

Of course the functions $\phi, a, b, h$ must be continuous nonnegative with $a, b$ continuously differentiable and nonincreasing in $y$ and $x$ respectively and $f$ must be Lipschitzian in its third argument. (See Ghoshal and Masood [2].) Note that Theorem 11.2.1 in Lakshmikantham and Leela [4] is not applicable in this situation. Further the applicability of the uniqueness theorem in Walter [6, p. 166] is also not immediately obvious for the problem.

\section{REFERENCES}

1. E. F. Beckenback and R. Bellman, Inequalities, Ergebnisse der Math., Springer-Verlag, Berlin and New York, 1961, p. 154.

2. S. Ghoshal and M. Abu. Masood, Gronwall's vector inequality and its application to a class of non-self adjoint linear and non-linear hyperbolic partial differential equations, J. Indian Math. Soc. 38 (1974), 383-394.

3. Jagdish Chandra and Paul W. Davis, Linear generalizations of Gronwall's inequality, Proc. Amer. Math. Soc. 60 (1976), 157-160.

4. V. Lakshmikantham and S. Leela, Differential and integral inequalities, Vol. II, Academic Press, New York, 1969, p. 224.

5. D. R. Snow, Gronwall's inequality for systems of partial differential equations in two independent variables, Proc. Amer. Math. Soc. 33 (1972), 46-54.

6. W. G. Walter, Differential and integral inequalities, Springer-Verlag, Berlin and New York, 1970.

Department of Mathematics and Statistics, Marathwada University, Aurangabad431004, INDIA 